\title{
A Novel Coupling between the Electron Structure and Properties of Perovskite Transition Metal Oxides
}

\author{
Ghous Narejo, Warren F. Perger \\ Electrical Engineering Department, Michigan Tech University, Houghton, USA \\ Email:wfp@mtu.edu
}

Received August 2, 2012; revised January 5, 2013; accepted January 12, 2013

Copyright (C) 2013 Ghous Narejo, Warren F. Perger. This is an open access article distributed under the Creative Commons Attribution License, which permits unrestricted use, distribution, and reproduction in any medium, provided the original work is properly cited.

\begin{abstract}
The ab-initio computational techniques are employed to extract the coupling between the electronic structure and magnetic properties for a wide variety of transition metal oxides. Optimized crystalline structures are computed by employing Hartree Fock (HF) and Density Functional Theory (DFT) techniques. The hydrostatic pressure is employed upon the optimized cubic crystalline structures of $\mathrm{BaScO}_{3}, \mathrm{BaTiO}_{3}, \mathrm{BaVO}_{3}, \mathrm{BaCrO}_{3}, \mathrm{BaMnO}_{3}$ and $\mathrm{BaFeO}_{3}$ to extract the coupling between the electronic structures and magnetic properties originating due to electron spin polarizations.
\end{abstract}

Keywords: Coupling; Spin; Strain

\section{Introduction}

The transition metals and their oxides are widely investigated by researchers [1-5]. The researchers employed the empirical, analytical and experimental methods [1-8] to extract the correlation between the electronic structures and properties of these materials. However, no major success has been reported in finding out the exact relationship between the crystalline structure and properties of these materials. We have attempted to investigate the complex nature of this interdependence existing in highly correlated physics of these materials. The complexity in these investigations arises due to an interaction between the electronic structures and properties. The latter are partially based upon the position of valence electrons in highly localized $d$-orbitals. This article explores the novel cubic perovskite phases for a wide variety of oxides of Sc-Fe. Later on, the interaction between these novel perovskite crystalline structures is compared with the computational results from other sources. An attempt has also been made to extract the interactions between the electronic structures and magnetic properties arising from electron spin polarization.

\section{Computational Methods and Paramters}

The Crystal09 code is employed to compute the exchange energy for the ferromagnetic and antiferromagnetic phases in each of the transition metal oxides. Fig- ures 1 and 2 show the periodic crystalline lattice of perovskite cubic $\mathrm{BaTiO}_{3}$ and $\mathrm{BaFeO}_{3}$ consisting of the supercells of an optimum size utilized during these computations. These super cells were employed to compute the optimized crystalline structure and its interaction with electronic properties arising out of the electron spin polarization. Hydrostatic strains are employed for a varying sizes of electronic structures around optimized crystalline volume for each perovskite. During these computations the electronic basis sets for $\mathrm{Ba}$ and $\mathrm{O}$ were kept same. for $\mathrm{BaScO}_{3}, \mathrm{BaVO}_{3}, \mathrm{BaCrO}_{3}$ and $\mathrm{BaMnO}_{3}$ to facilitate the SCF convergence.

The computational results are reported in Tables 1-5 and shown in Figures 1-8. The optimized crystalline structure is compared with experiemental values if available and four additional computations of electronic structures and properties are performed. The hydrostatic compression or expansion of each crystalline structure is achieved by employing expansions and reductions of volume in small increments.

In this way, five separate computations are done for each of the crystalline systems. These computations are repeated for a large variety of crystalline systems to check the consistency.

\section{Model}

Some researchers $[5,7,8]$ have proposed working theoretical models for a wide variety of transition metal oxides 


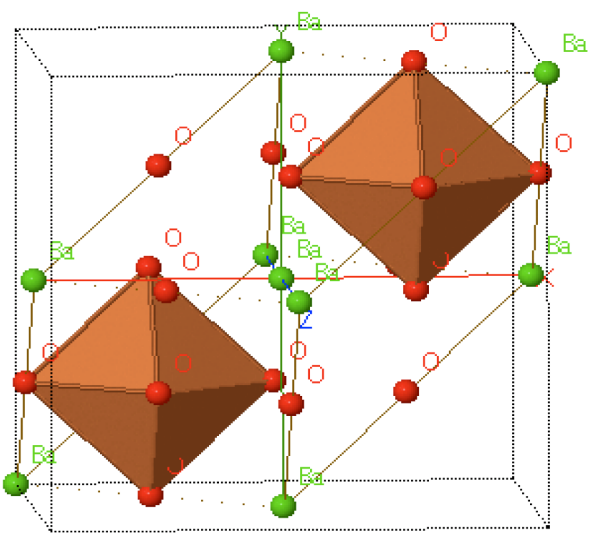

Figure 1. An octahedral is formed by $\mathrm{O}$ atoms having $\mathrm{Fe}$ atom in the middle. Green and red color spheres represent $\mathrm{Ba}, \mathrm{O}$ and $\mathrm{Fe}$ atoms could not be seen as these are positioned in the middle of each cage in a perovskite $\mathrm{BaFeO}_{3}$.

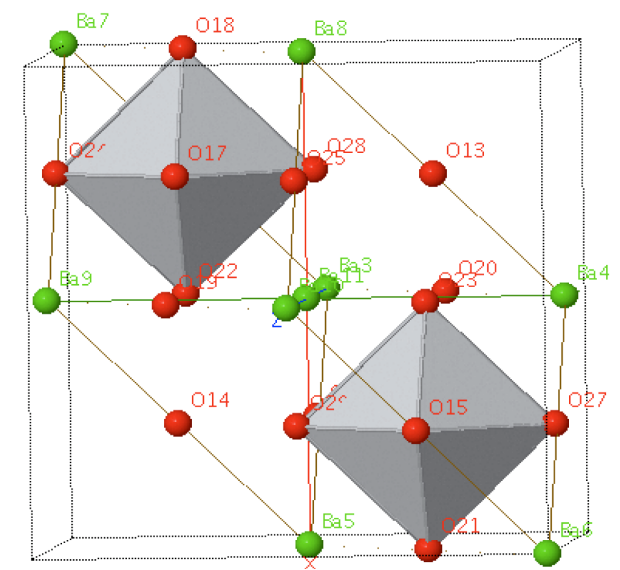

Figure 2. An octahedral is formed by $\mathrm{O}$ atoms having $\mathrm{Fe}$ atom in the middle. Green and red color spheres represent $\mathrm{Ba}$ and $\mathrm{O}$ while grey colored $\mathrm{Ti}$ atoms are positioned in the middle of each cage in a perovskite $\mathrm{BaTiO}_{3}$.

Table 1. Computations of $E_{f m}, E_{e x c h}^{f m}, E_{a f m}$ and $E_{e x c h}^{a f m}$ are done for lattice constant $a$ in cubic $\mathrm{BaScO}_{3}$. The units of energy are in Hartree.

\begin{tabular}{cccc}
\hline$a(\AA)$ & $E_{\text {exch }}^{\text {fm }}$ & $E_{\text {exch }}^{\text {afm }}$ & $\delta$ \\
\hline 4.120 & -132.8511 & -132.6138 & -0.2373 \\
& -132.8497 & -132.6086 & -0.2411 \\
4.125 & -132.8408 & -132.6031 & -0.2377 \\
& -132.8394 & -132.5964 & -0.243 \\
4.13 & -132.8305 & -132.5923 & -0.2382 \\
& -132.8297 & -132.5860 & -0.2437 \\
4.135 & -132.8211 & -132.5817 & -0.2394 \\
& -132.8194 & -132.5749 & -0.2455 \\
4.140 & -132.8077 & -132.5690 & -0.2387 \\
& -132.8094 & -132.5657 & -0.2437 \\
\hline
\end{tabular}

Table 2. Computations of $E_{f m}, E_{e x c h}^{f m}, E_{a f m}$ and $E_{e x c h}^{a f m}$ are done for lattice constant $a$ in cubic $\mathrm{BaTiO}_{3}$. The units of energy are Hartree.

\begin{tabular}{cccc}
\hline$a(\AA)$ & $E_{\text {exch }}^{\text {fm }}$ & $E_{\text {exc }}^{\text {afm }}$ & $\delta$ \\
\hline 4.00 & -138.4905 & -138.7541 & 0.2636 \\
& -138.7533 & -138.7532 & -0.0001 \\
4.005 & -138.4785 & -138.7403 & 0.2618 \\
& -138.7396 & -138.7394 & -0.0002 \\
4.01 & -138.4663 & -138.7265 & 0.2602 \\
& -138.7259 & -138.7125 & -0.0134 \\
4.015 & -138.4546 & -138.7132 & 0.2586 \\
& -138.4554 & -138.7125 & 0.2571 \\
4.02 & -138.4427 & -138.6996 & 0.2569 \\
& -138.6990 & -138.6989 & 0.0001 \\
\hline
\end{tabular}

Table 3. Computations of $E_{f m}, E_{e x c h}, E_{a f m}$ and $E_{e x c h}$ are done for lattice constant $a$ in cubic $\mathrm{BaVO}_{3}$. The units of energy are Hartree.

\begin{tabular}{cccc}
\hline$a(\AA)$ & $E_{\text {exch }}^{\text {fm }}$ & $E_{\text {exch }}^{\text {afm }}$ & $\delta$ \\
\hline 4.049 & -145.2346 & -143.9615 & -1.2731 \\
& -145.0421 & -143.9616 & -1.0805 \\
4.054 & -145.2010 & -143.9488 & -1.2522 \\
& -145.2075 & -143.9489 & -1.2586 \\
4.059 & -145.2231 & -143.9360 & -1.2871 \\
& -145.3132 & -143.9362 & -1.377 \\
4.064 & -145.1868 & -143.9234 & -1.2634 \\
& -145.1926 & -143.9235 & -1.2691 \\
4.069 & -145.1994 & -143.9108 & -1.2866 \\
& -145.1770 & -143.9110 & -1.266 \\
\hline
\end{tabular}

Table 4. Computations of $E_{f m}, E_{\text {exch }}^{f m}, E_{\text {afm }}$, and $E_{\text {exch }}^{a f m}$ are done for lattice constant $a$ in cubic $\mathrm{BaCrO}_{3}$. The units of energy are Hartree.

\begin{tabular}{cccc}
\hline$a(\AA)$ & $E_{\text {exch }}^{\text {m }}$ & $E_{\text {exch }}^{\text {afm }}$ & $\delta$ \\
\hline 3.8436 & -151.8259 & -150.3628 & -1.4631 \\
& -151.8267 & -150.3763 & \\
3.8486 & -151.8586 & -150.3475 & -1.5111 \\
& -151.8283 & -150.3609 & \\
3.8536 & -151.9614 & -150.3326 & -1.6288 \\
& -151.8653 & -150.3381 & \\
3.8586 & -151.8034 & -150.3179 & -1.4855 \\
& -151.7902 & -150.3232 & \\
3.8636 & -151.7371 & -150.3032 & -1.4339 \\
& -151.8065 & -150.3085 & \\
\hline
\end{tabular}


Table 5. Computations of $E_{f m}, E_{e x c h}^{f m}, E_{a f m}$ and $E_{e x c h}^{a f m}$ are done for lattice constant $a$ in cubic $\mathrm{BaMnO}_{3}$. The units of energy are Hartree.

\begin{tabular}{cccc}
\hline$a(\AA)$ & $E_{\text {exch }}^{\text {fm }}$ & $E_{\text {exch }}^{\text {afm }}$ & $\delta$ \\
\hline 3.80 & -34.8595 & -157.2687 & 122.4092 \\
& -156.8650 & -157.2736 & \\
3.85 & -65.2846 & -156.8965 & 91.6119 \\
& -158.2801 & -156.9355 & \\
3.90 & -159.0151 & -156.8844 & -2.1307 \\
& -157.9015 & -157.2532 & \\
3.95 & -64.0915 & -157.2102 & 93.1187 \\
& -157.5813 & -156.9111 & \\
4.00 & -159.0042 & -157.2003 & -1.8039 \\
& -157.6340 & -156.8992 & \\
\hline
\end{tabular}

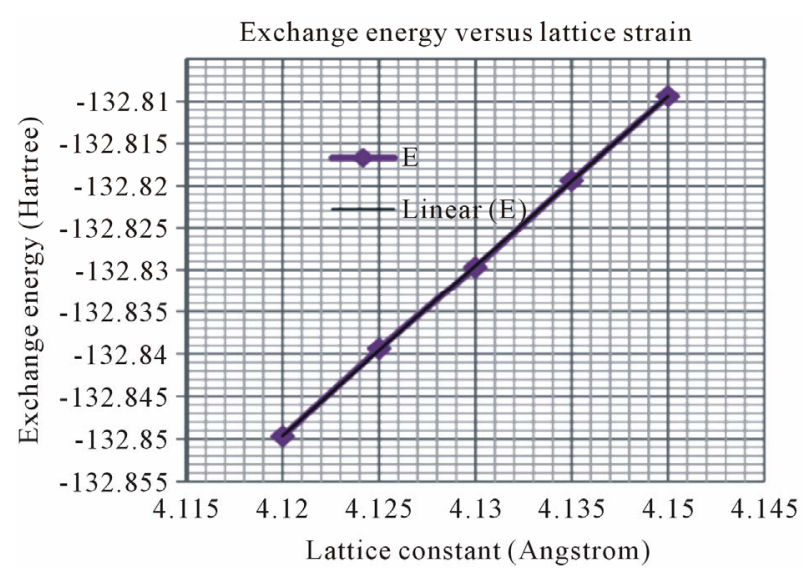

Figure 3. Exchange energy vs. lattice strain for cubic $\mathrm{BaScO}_{3}$. A decrease in exchange energy can be seen for the compression of lattice. The straight line is drawn to signify the linearity of the trend.

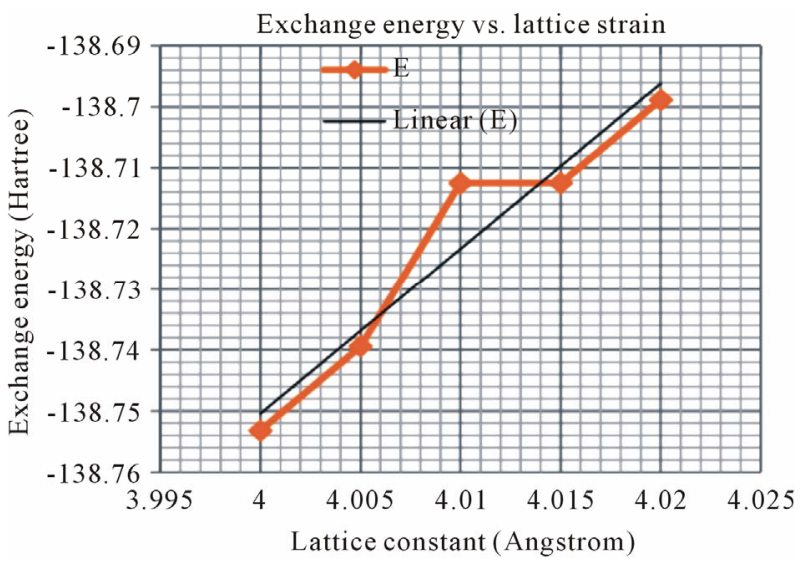

Figure 4. Exchange energy vs. lattice strain for cubic $\mathrm{BaTiO}_{3}$. A decrease in exchange energy can be seen for the compression of lattice. There is smaller deviation from the linear trend.

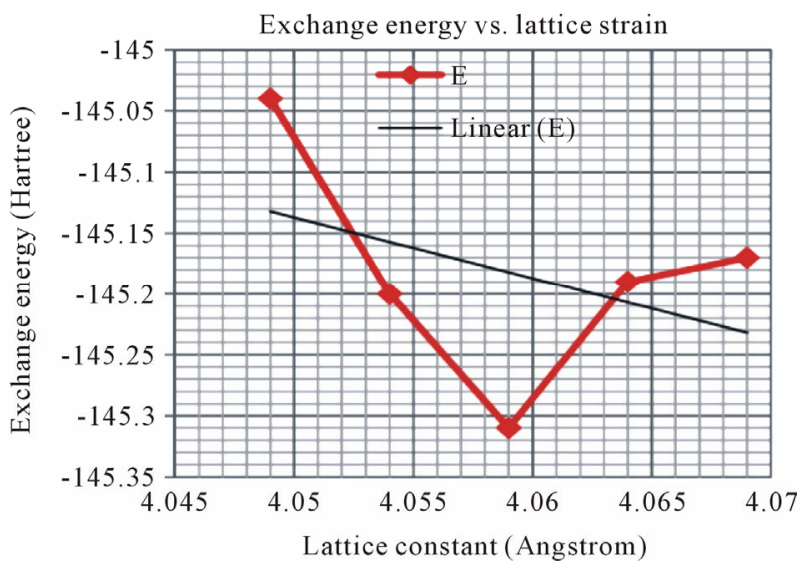

Figure 5. Exchange energy vs. lattice strain for cubic $\mathrm{BaVO}_{3}$. A slight increase in the exchange energy can be seen for the compression of lattice. The nonlinear dependence of the exchange energy on lattice strain is more pronounced.

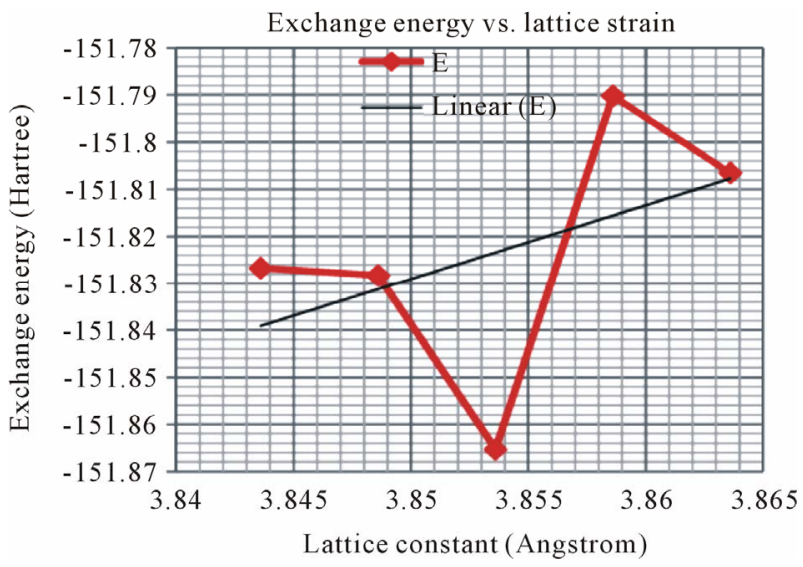

Figure 6. Exchange energy vs. lattice strain for cubic $\mathrm{BaCrO}_{3}$. A decrease in exchange energy can be seen for the compression of lattice. The oscillatory character of exchange energy is also persistent.

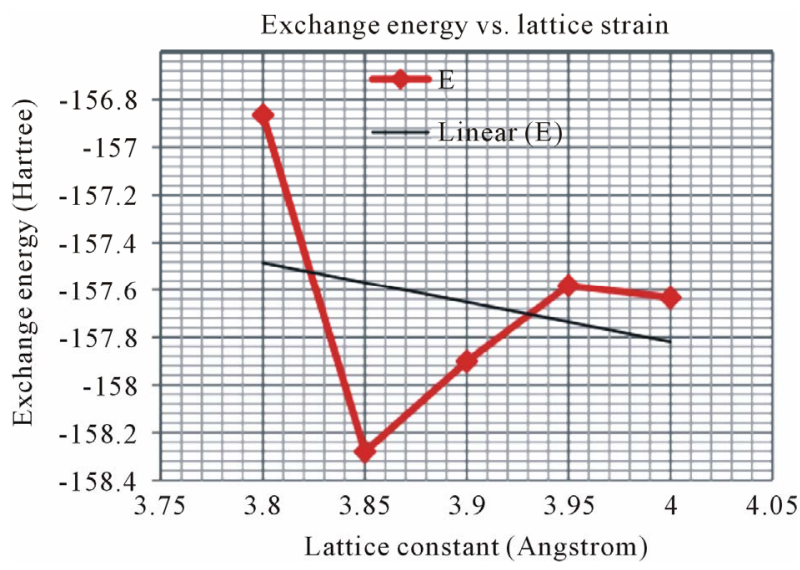

Figure 7. Exchange energy vs. lattice strain for cubic $\mathrm{BaMnO}_{3}$. The nonlinear dependence of the exchange energy on the compression of lattice can be observed. Exchange energy has slightly increased with the compression of the lattice in this case. 


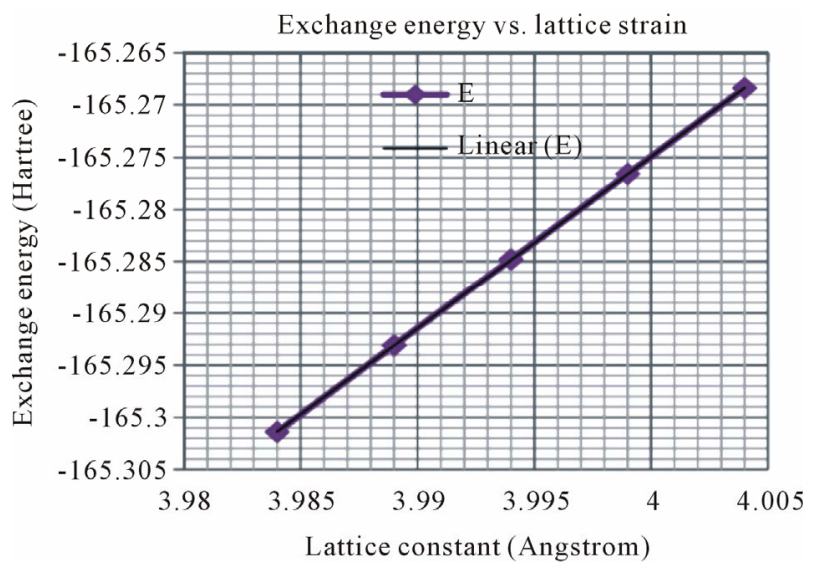

Figure 8. Exchange energy vs. lattice strain for cubic $\mathrm{BaFeO}_{3}$. A decrease in exchange energy can be seen for the compression of lattice showing a linear dependence on the strain.

known as manganites perovskites. The crystalline geometry and properties of cubic $\mathrm{BaMnO}_{3}$ may be well suited to the quantum mechanical model. These models advocate a delicate balance between the crystalline field and Hund's pairing energy. The crystalline field in these models originates due to a coulombic force between the electrons and atomic centers. The electrostatic fields partially attributed to these crystalline fields are intricately interdependent. The interaction between the crystalline field and Hund's pairing energy for the relaxed as well as the strained crystalline structures is interpreted from this model here. The phenomenon of ferromagnetic spin exchange, depending upon the highly correlated electrons in a crystal field, is also accommodated in the model. It is assumed that the ferromagnetic or antiferromagnetic properties due to electron spin polarization depend upon the crystal field of the strained lattice structure. This model can also be applied to manganites $\left(\mathrm{AMO}_{3}\right)$, titanates $\left(\mathrm{ATiO}_{3}\right)$ and vanadates $\left(\mathrm{AVO}_{3}\right)$ as the crystal field splitting is predominant and is relevant in all the material systems discussed as in ref. [7].

The Hamiltonian for a typical transition metal oxide may be

$$
H_{\text {eff }}=H_{\text {hund }}+H_{t_{2 g}}+H_{e_{g}}
$$

The $H_{e_{g}}$ term in Equation (1) may expresse the energy component due to $e_{g}$ valence electrons of the transition metals $\sigma$-bonded with the $p$-valence electrons for $\mathrm{O}$ atoms in placed in an octahedral complex. The $H_{t_{2 g}}$ term expresses the energy component due to $t_{2 g}$ electron which are $\pi$-bonded the $p$-electrons of $\mathrm{O}$ atoms.

The term $H_{\text {hund }}$ expresses the Hund energy for electrons

$$
H_{\text {hund }}=J_{H} \sum_{i} S_{i} S_{i}^{t 2 g}
$$

The second energy component in Equation (1) is at- tributed to electrons localized at $t_{2 g}$ suborbital

$$
H_{t_{2 g}}=J_{i j} S_{i}^{t 2 g} S_{j}^{t 2 g}
$$

Equation (4) splits the $H_{e_{g}}$ term further

$$
H_{e_{g}}=\Delta \sum_{i} L_{i z}+\sum_{\langle i j\rangle \sigma \gamma^{\prime}} t_{\gamma}^{i j} \gamma^{\prime}\left(a_{i \gamma \sigma}^{\dagger} a_{j \gamma^{\prime} \sigma}\right)+\sum_{\beta} H_{U_{\beta}}
$$

In Equation (4), the subscripts $i$ and $j$ express the nearest neighbors on ionic sites, $a_{i \gamma \sigma}^{\dagger}$ and $a_{j \gamma^{\prime} \sigma}$ are the creation and annihilation operators respectively. The term $t$ in Equation (4) expresses the kinetic energy of $e_{g}$ electrons in $\mathrm{BaMnO}_{3}, \mathrm{BaCrO}_{3}$ and $\mathrm{BaFeO}_{3}$ and electrostatic energy term $U$. The Equation (4) takes into account the kinetic energy of electrons delocalized due to strains on the $\sigma$-bonded $e_{g}$ and $p$-orbitals. The electrons hop between the cation and anion sites termed as $i$ and $j$.

$$
\begin{gathered}
t=\sum_{\langle i j\rangle \sigma \gamma \gamma^{\prime}} t_{\gamma}^{i j} \gamma^{\prime}\left(a_{i \gamma \sigma}^{\dagger} a_{j \gamma^{\prime} \sigma}\right) \\
t_{i j}^{\gamma \gamma^{\prime}}=\alpha_{\gamma \gamma^{\prime}} t_{o i j}
\end{gathered}
$$

The electrostatic energy term $U$ expresses the on-site electron correlation in transition metal cations resulting in the electron localization on transition metal sites. The symbol $t$ in Equation (5) is the hopping integral for electrons transferred under the action of strains between ions $i$ and the nearest neighbors $j$. Hund's energy consists of energy components due to $t_{2 g}$ and $e_{g}$ electrons which are well-localized on each transition metal site due to electron correlations as shown in Equation (7).

$$
J_{H}=H_{U}+H_{U_{t_{2 g}}}+H_{U_{e_{g}}}
$$

It is assumed that the term $\Delta \varepsilon$ in Equation (8) may be related with the change in the crystal field energy originating due to external strain. The term $\sum_{i} T_{i}$ expresses the change in the orbital spin angular momentum due to the effect of strain.

$$
\Delta \varepsilon=\Delta \sum_{i} T_{i}
$$

\section{Results and Discussion}

The computational results are shown in Tables 1-5 and Figures 3-5. It is observed that the computational values of energy due to spin polarizations are coupled with electronic structures. The results are also plotted in Figures 3-5. The oscillations in energy for the $\mathrm{BaVO}_{3}$, $\mathrm{BaCrO}_{3}$ and $\mathrm{BaMnO}_{3}$ show that the coupling between the electronic structures and valence electrons are fairly complex. In Tables 1-5, the $E_{f m}, E_{\text {exch }}^{f m}, E_{a f m}$ and $E_{\text {exch }}^{a f m}$ represent the energies arising from ferromagnetic and antiferromagnetic spin polarizations.

The primary effect of the external pressure on the transition metal oxides is to compress or expand their 
bond lengths connecting the transition metal and oxygen atoms in a perovskite. The expansion and contraction of the bond length result in the weakening or strengthening of the electron interactions within crystal structures among the transition metal $e_{g}$ electrons and oxygen $p$ electrons. The interactions between electrons and ions couple them in a complicated manner.

Moreover, these interactions are facilitated by the strains only if there are enough numbers of electrons in $e_{g}$ valence orbitals. This phenomenon can be observed in the computational results obtained for $\mathrm{BaCrO}_{3}, \mathrm{BaMnO}_{3}$ and $\mathrm{BaFeO}_{3}$. Less variations in energy as a function of lattice strains for some oxides is a function of the localized nature of the $t_{2 g}$ and $e_{g}$ electron orbitals.

The oxides of transition metal have varied number of electrons in their highly correlated $d$-orbitals. The contracted wavefunctions of $d$ electrons in $\mathrm{BaScO}_{3}, \mathrm{BaTiO}_{3}$, $\mathrm{BaVO}_{3}, \mathrm{BaMnO}_{3}$ and $\mathrm{BaFeO}_{3}$ experience the varied degree of competitive forces of the coulomb repulsion versus hybridization. The former tries to localize the electrons at atomic lattice sites while the latter favors the overlaps with $p$ - and $d$-orbitals of $\mathrm{O}$ and transition metal to delocalize these electrons. The forces of coulomb repulsion and hybridization are varied by lattice strain. A trend can be seen in all computations as there is a consistent decrease in energy for the compression and increase in energy for expansion of lattice volume. The chemical bond in transition metal oxides is a combination of covalent and ionic parts. The covalent and ionic parts vary as the transition metal ionic radius increases from $\mathrm{Sc}$ to $\mathrm{Fe}$. The contribution of ionic bonding is increased as the number of electrons in transition metals are increased with more impact on the energy as a function of lattice strain. The computational results of $\mathrm{BaScO}_{3}, \mathrm{BaTiO}_{3}, \mathrm{BaVO}_{3}, \mathrm{BaCrO}_{3}, \mathrm{BaFeO}_{3}$ show significant variations in chemical bonding from strongly covalent to moderately ionic in nature for the materials tested.

From the computed results shown in Tables 1-6, an increase in the energy is observed for spins polarized in same direction for all crystalline systems tested confirming the coupling between the crystalline structure and electron spin polarization.

\section{Concluding Remarks}

We have employed first principles computations to extract the coupling between the crystalline structure and electron spin polarization. The optimized crystalline structures are computed by a variety of methods for each of the transition metal oxides. Later on, the coupling between the electronic structure and electron spin polarization is determined by computing the energy for the spins aligned in the parallel as well as antiparallel polariza-
Table 6. Computations of $E_{f m}, E_{\text {exch }}^{f m}, E_{a f m}$, and $E_{e x c h}^{a f m}$ are done for lattice constant $a$ in cubic $\mathrm{BaFeO}_{3}$. The units of energy are Hartree.

\begin{tabular}{cccc}
\hline$a(\AA)$ & $E_{\text {exch }}^{\text {fm }}$ & $E_{\text {exch }}^{\text {ofm }}$ & $\delta$ \\
\hline 3.984 & -165.3002 & -164.6210 & -0.6792 \\
& -165.3014 & -164.6329 & -0.6685 \\
3.989 & -165.2919 & -164.6123 & -0.6796 \\
& -165.2931 & -164.6254 & -0.6677 \\
3.994 & -165.2836 & -164.6046 & -0.679 \\
& -165.2848 & -164.6173 & -0.6675 \\
3.999 & -165.2754 & -164.5965 & -0.6789 \\
& -165.2766 & -164.6092 & -0.6674 \\
4.004 & -165.2673 & -164.5896 & -0.6777 \\
& -165.2684 & -164.6020 & -0.6664 \\
\hline
\end{tabular}

tions. It is observed that the compression of the the bulk crystal results in the lowering of the the energy confirming the fact that the former is intricately coupled with the latter.

It is seen that the coupling between the electronic structure and electronic polarization varies with the occupation of electrons in the outermost orbitals. During computations, it has been observed that the compression lowers the electron energy in the transition metal oxides of Sc-Fe. The lowering of the polarization energy may be attributed to the stronger coupling between transition metal $e_{g}$ and $\mathrm{O} p$-orbitals forming a $\sigma$-bond.

\section{Acknowledgements}

One of the authors (WFP) gratefully acknowledges the support of the Office of Naval Research Grant N0001401-1-0802 through the MURI program.

\section{REFERENCES}

[1] R. Gopalan and V. Chandrasekharan, "Room temperature multiferroism and magnetoelectric coupling in $\mathrm{BaTiO}_{3}$ $\mathrm{BaFe}_{12} \mathrm{O}_{19}$ System Srinivas," Solid State Communications, Vol. 149, No. 9-10, 2009, pp. 367-370.

[2] J. P. Velev, P. A. Dowben, E. Y. Tsymbal, S. J. Jenkin, and A. Caruso, "Interface Effects in Spin-Polarized Metal/Insulator Layered Structures," Surface Science Reports, Vol. 63, No. 9, 2008, pp. 400-425. doi:10.1016/j.surfrep.2008.06.002

[3] J. B. Goodenough, "Electron-Lattice Interactions in Manganese-Oxide," Perovskites, Fundamental Materials Research, 2002, pp. 127-133.

[4] C. N. R. Rao, "Transition Metal Oxides," Annual Review of Physical Chemistry, Vol. 40, 1989, pp. 291-326. 
doi:10.1146/annurev.pc.40.100189.001451

[5] S. Horiuchi, Y. Okimoto, R. Kumai and Y. Tokura, "Quantum Phase Transition in Organic Charge-Transfer Complexes," Science, Vol. 299, 2003.

[6] J. P. Velev, C.-G. Duan, J. D. Burton, A. Smogunov, M. K. Niranjan, E. Tosatti, S. S. Jaswal and E. Y. Tsymbal, "Magnetic Tunnel Junctions with Ferroelectric Barriers: Prediction of Four Resistance States from First Princi- ples," Nano Letters, Vol. 9, No. 1, 2009, pp. 427-432. doi:10.1021/n1803318d

[7] I. Sumio, O. Satoshi and M. Sadamichi, Physics of Transition Metal Oxides, 1997.

[8] Y. Tokura and N. Nagaosa, "Orbital Physics in Transition-Metal Oxides," Science, Vol. 288, No. 462, 2000, pp. 462-468. 